\title{
Hepatocellular carcinoma and liver transplant: beyond the Milan criteria and the risk of "short-blanket" syndrome
}

\author{
Stefano Gitto ${ }^{1 \#}$, Stefano Di Sandro ${ }^{2 \#}$, Paolo Magistri ${ }^{2}$, Pietro Andreone ${ }^{3}$, Fabrizio Di Benedetto ${ }^{2}$ \\ ${ }^{1}$ Department of Experimental and Clinical Medicine, University of Florence, Florence, Italy; ${ }^{2}$ Hepato-Pancreato-Biliary Surgery and Liver \\ Transplantation Unit, ${ }^{3}$ Internal and Metabolic Medicine, AOU di Modena and University of Modena and Reggio Emilia, Modena, Italy \\ \#These authors contributed equally to this work. \\ Correspondence to: Pietro Andreone. Internal and Metabolic Medicine, AOU di Modena and University of Modena and Reggio Emilia, Modena \\ 41126, Italy. Email: pietro.andreone@unimore.it. \\ Provenance and Peer Review: This article was commissioned by the editorial office, Hepatobiliary Surgery and Nutrition. The article did not undergo \\ external peer review. \\ Comment on: Mehta N, Dodge JL, Grab JD, et al. National experience on down-staging of hepatocellular carcinoma before liver transplant: influence \\ of tumor burden, alpha-fetoprotein, and wait time. Hepatology. 2020;71:943-54.
}

Submitted Nov 06, 2019. Accepted for publication Nov 21, 2019.

doi: 10.21037/hbsn.2019.11.34

View this article at: http://dx.doi.org/10.21037/hbsn.2019.11.34

Liver transplant (LT) is considered the best option for patients with hepatocellular carcinoma (HCC) confined into the liver and generally associated with liver cirrhosis (1). The Milan criteria (MC) represent a milestone in the selection of these patients (2). Therefore, basing on MC, the 5 -year survival rate after LT could increase to $70 \%$, with a HCC recurrence rate lower than $10 \%$ (2). Despite that, too many patients affected by HCC may not benefit from LT due to a tumor stage beyond the selection criteria. Over the last two decades, authors and physicians tried to overcome the MC exploring the opportunity to safely transplant patients beyond (3). Notably, the donor shortage and the competition between patients listed due to HCC and those listed for other indications, force physicians in selecting candidates with the best transplant benefit (3).

Nowadays, the majority of patients listed for LT receive locoregional treatments (LTRs) for HCC with the aim to control the tumor progression waiting for the LT and to improve post-LT outcome (4). Furthermore, LRTs allow down staging the tumor burden at diagnosis with the possibility to transplant patients unsuitable for LT at the first tumor evaluation (4).

Although the MC remains a strong predictor of postLT survival, many patients showed good or poor postLT outcome in spite attending or not those criteria. The strongest evidence, which has recently affirmed, demonstrates that the biological tumor behaviour may impair results even in patients with safe morphological criteria (tumor size and number). By this way, the relevance of the alpha-fetoprotein and tumor response to the LRTs has significantly grown up. Indeed, the most recent staging systems include the consideration of tumor biology's clues such as the progression after LRTs or the absolute value and trend of the alpha-fetoprotein before transplant (5-7).

Accordingly, the Metroticket 2.0 is one of the most accurate models able to predict post-LT HCC related survival basing on tumor number and size and alphafetoprotein evaluated at the last re-staging time (5).

On 2016, Mazzaferro et al. (8) proposed a philosophical framework about the transplantable tumor (TT) including all the pre-operative variables related to the tumor stage, opportunity of downstaging, and response to the LRTs before the transplant. TTs were classified into 8 stages with progression from lower stage with fewer priorities to the transplant until the highest stage with highest priority. A recent study tried to validate the prediction power of this staging system on a real patient cohort, exploring the correlation between the stages and the dropout risk before LT and the correlation between tumor stages and risk of post-LT HCC related death (9). Therefore, the TT staging appeared predictive for both higher and lower risk classes. However, it resulted inaccurate for the intermediate classes. Indeed, patients classified at intermediate risk appeared 
having benefit from a delayed transplant (longer than 2 months since the last tumor staging) (9). The message to delay a LT in view to improve the post-LT patient survival should be taken with caution. Although selected patients may benefit of a postponed transplant, in term of overall survival and specific tumor survival compared to an earlier transplant, the risk of patient drop-out waiting for a delayed transplant may be the really worst scenario for the patient himself.

Mehta et al. (10) recently approached this complex issue with a well-designed national study. Authors retrospectively analysed data from United Network for Organ Sharing (UNOS) database about LT for HCC between 2012 and 2015. Candidates were classified into the following subgroups: (I) "Milan criteria" group (patients with HCC continuously within homonym criteria); (II) "UNOS-DS" group (UNOS down-staging inclusion criteria: 1 lesion $>5$ and $\leq 8 \mathrm{~cm}, 2-3$ lesions at least one $>3$ and $\leq 5 \mathrm{~cm}$ with total tumor diameter $\leq 8 \mathrm{~cm}$, or $4-5$ lesions each $\leq 3 \mathrm{~cm}$ with total tumor diameter $\leq 8 \mathrm{~cm}$ ); (III) "AC-DS" group (initial tumor burden exceeding UNOS-DS inclusion criteria with no upper burden limit).

Notably, the subgroups 2 included patients selected according to UNOS-DS criteria adopted by 2017. The same authors already tested these standards in a multi-centre study with brilliant 5 -year post-LT survival (80\%) (11).

Authors subdivided the UNOS regions according to the median time from listing to LT [ $>9$ months waitlist times was long wait region (LWR), 3-9 months mid wait region (MWR), and $<3$ months was considered short wait region (SWR), respectively] and registered the alpha-fetoprotein (AFP) at the time of LT.

Authors analysed data from 3,819 patients $(85.8 \%$ were in the first subgroup, $11.0 \%$ in the second, $3.2 \%$ in the third one). The 3 -year cumulative post-LT survival was $83.2 \%$ in the Milan group, $79.1 \%$ in the UNOS-DS group ( $\mathrm{P}=0.17$ vs. Milan), and $71.4 \%$ in the AC-DS group ( $\mathrm{P}=0.04 v s$. Milan). Indeed, the Milan and UNOS-DS groups showed the same 3 -year survival. However, only in the second and third subgroups, substantial differences in post-LT survival were detected according to the waiting time region. In UNOS-DS group, the 3-year post-LT survival was lower in MWR (72.5\%) and SWR (78.7\%) compared to LWR (92.3\%) $(\mathrm{P}=0.009)$ with the same (but not statistically significant) trends for AC-DS group (LWR: 93.3\%, MWR: 65.7\%, SWR: 73.0\%).

Post-LT HCC recurrence was observed in $4.4 \%$ of patients of Milan groups $v s .9 .2 \%$ and $10.7 \%$ of the others two $(\mathrm{P}<0.001)$.

Then, authors conducted univariate and multivariate analysis for searching predictors of post-LT survival and HCC recurrence. Notably, authors considered only subgroups at major risk of worse outcome (subgroups 2 and 3). Authors demonstrated that short or mid permanence on waitlist (with consequent short or mid cancer observation before LT) together with AFP $>100 \mathrm{ng} / \mathrm{mL}$, strongly correlate with worse post-LT survival. Furthermore, they reported that AFP $>100 \mathrm{ng} / \mathrm{mL}$ was the only independent predictor of post-LT HCC recurrence.

Data from this study clearly indicate the significance of acquiring a minimal observation time to establish the effectiveness of down-staging (DS) approach. At the same time, AFP level seems to have a chief role in stratifying the risk of HCC recurrence. In this regard, authors used this study to validate the risk estimation of tumor recurrence after transplant (RETREAT) score, suitable for predicting post-LT HCC recurrence in the DS populations. Increasing RETREAT scores not only predicted increased postLT HCC recurrence but worse post-LT survival too. Remarkably, RETREAT score computes AFP at LT, microvascular invasion, and the sum of the largest viable tumor plus number of viable tumors on explant (12).

Although the study demonstrates important results concerning the correlation between HCC DS and results after LT, some limitations oblige to carefully consider the final messages. First, the correlation between the pretransplant HCC management and post-LT results should be designed by an intention-to-treat point of view, basing on the necessity to balance the risk of patient drop-out before the transplant and the post-LT survival or HCC specific survival. The effect of the time-to-transplant, fast or slow between listing and transplant, and the patient survival should take into account how many patients have lost the chance to be transplanted because of tumor progression. The different survival reported by Mehta and co-workers among SWR, MWR, and LWR may be re-balanced after an intentionto-treat analysis and this is mandatory. Secondly, although the large collected series and the accurate study methods, the median follow-up of 1.9 years appears significantly short to draw grounded conclusions with risk of HCC recurrence's underestimation. Moreover, the RETREAT score has strongly demonstrated its validity as predictive tool of HCC recurrence after transplant. However, the scientific community has recently concentrated its attention on the pre-transplant variables, with the aim to obtain information as much as possible before LT, rather than after. Indeed, despite the accurate radiological restaging since all the group 2 and 3 patients resulted attending the transplant criteria, 
$32.5 \%$ and $40.5 \%$ of patients were outside the criteria at the explant tumor staging. By the Mehta's study (10) we could not understand the interval between the last re-staging and the transplant, thus it results difficult distinguishing between a radiological tumor underestimation or tumor progression in the time between last re-staging and transplant.

The reproducibility of the study results appears difficult since the stratification of wait time is not based on individual variables but on the UNOS regions.

Other open question regards the possible use of AFP for the selection of candidates. This study (10), but also others $(5,7)$, underlined the relevance of AFP for improving the selection criteria in terms of $\mathrm{HCC}$ recurrence prediction. However, we well know that $30-40 \%$ of HCC are diagnosed with normal serum AFP (13) and this might represent a potential limitation for the clinical application.

Furthermore, other tools for a better selection of LT candidates should be evaluated such as alternative serum markers [e.g., lens culinaris-agglutinin-reactive fraction of AFP (AFP-L3) and protein induced by vitamin $\mathrm{K}$ absence or antagonist-II (PIVKA-II)] (14) and/or hepatic gene signature useful for detection of more aggressive cancer (15).

\section{Acknowledgments}

Funding: None.

\section{Footnote}

Conflicts of Interest: All authors have completed the ICMJE uniform disclosure form (available at http://dx.doi. org/10.21037/hbsn.2019.11.34). The authors have no conflicts of interest to declare.

Ethical Statement: The authors are accountable for all aspects of the work in ensuring that questions related to the accuracy or integrity of any part of the work are appropriately investigated and resolved.

Open Access Statement: This is an Open Access article distributed in accordance with the Creative Commons Attribution-NonCommercial-NoDerivs 4.0 International License (CC BY-NC-ND 4.0), which permits the noncommercial replication and distribution of the article with the strict proviso that no changes or edits are made and the original work is properly cited (including links to both the formal publication through the relevant DOI and the license). See: https://creativecommons.org/licenses/by-nc-nd/4.0/.

\section{References}

1. European Association for the Study of the Liver. EASL clinical practice guidelines: liver transplantation. J Hepatol 2016;64:433-85.

2. Mazzaferro V, Regalia E, Doci R, et al. Liver transplantation for the treatment of small hepatocellular carcinomas in patients with cirrhosis. N Engl J Med 1996;334:693-9.

3. Toso C, Mazzaferro V, Bruix J, et al. Toward a better liver graft allocation that accounts for candidates with and without hepatocellular carcinoma. Am J Transplant 2014;14:2221-7.

4. Cescon M, Cucchetti A, Ravaioli M, et al. Hepatocellular carcinoma locoregional therapies for patients in the waiting list. Impact on transplantability and recurrence rate. J Hepatol 2013;58:609-18.

5. Mazzaferro V, Sposito C, Zhou J, et al. Metroticket 2.0 model for analysis of competing risks of death after liver transplantation for hepatocellular carcinoma. Gastroenterology 2018;154:128-39.

6. Yao FY, Ferrell L, Bass NM, et al. Liver transplantation for hepatocellular carcinoma: expansion of the tumor size limits does not adversely impact survival. Hepatology 2001;33:1394-403.

7. Duvoux C, Roudot-Thoraval F, Decaens T, et al. Liver transplantation for hepatocellular carcinoma: a model including $\alpha$-fetoprotein improves the performance of Milan criteria. Gastroenterology 2012;143:986-94.e3; quiz e14-5.

8. Mazzaferro V. Squaring the circle of selection and allocation in liver transplantation for HCC: an adaptive approach. Hepatology 2016;63:1707-17.

9. Di Sandro S, Bagnardi V, Cucchetti A, et al. From a philosophical framework to a valid prognostic staging system of the new "comprehensive assessment" for transplantable hepatocellular carcinoma. Cancers (Basel) 2019. doi: $10.3390 /$ cancers 11060741.

10. Mehta N, Dodge JL, Grab JD, et al. National experience on down-staging of hepatocellular carcinoma before liver transplant: influence of tumor burden, alpha-fetoprotein, and wait time. Hepatology. 2020;71:943-54.

11. Mehta N, Guy J, Frenette CT, et al. Excellent outcomes of liver transplantation following down-staging of hepatocellular carcinoma to within Milan criteria: a multicenter study. Clin Gastroenterol Hepatol 2018;16:955-64.

12. Mehta N, Heimbach J, Harnois DM, et al. Validation of a risk estimation of tumor recurrence after transplant (RETREAT) score for hepatocellular carcinoma recurrence after liver transplant. JAMA Oncol 2017;3:493-500.

13. Johnson PJ. Role of alpha-fetoprotein in the diagnosis and 
management of hepatocellular carcinoma. J Gastroenterol Hepatol 1999;14 Suppl:S32-6.

14. Park SJ, Jang JY, Jeong SW, et al. Usefulness of AFP, AFP-L3, and PIVKA-II, and their combinations in diagnosing hepatocellular carcinoma. Medicine (Baltimore) 2017;96:e5811.

15. Villa E, Critelli R, Lei B, et al. Neoangiogenesis-related genes are hallmarks of fast-growing hepatocellular carcinomas and worst survival. Results from a prospective study. Gut 2016;65:861-9.

Cite this article as: Gitto S, Di Sandro S, Magistri P, Andreone P, Di Benedetto F. Hepatocellular carcinoma and liver transplant: beyond the Milan criteria and the risk of "shortblanket” syndrome. HepatoBiliary Surg Nutr 2020;9(4):518521. doi: 10.21037/hbsn.2019.11.34 